Document downloaded from:

http://hdl.handle.net/10251/154999

This paper must be cited as:

Peng, Y.; Albero-Sancho, J.; García Gómez, H. (2019). Surface Silylation of Hybrid Benzidinium Lead Perovskite and its Influence on the Photocatalytic Activity. ChemCatChem. 11(24):6384-6390. https://doi.org/10.1002/cctc.201901681

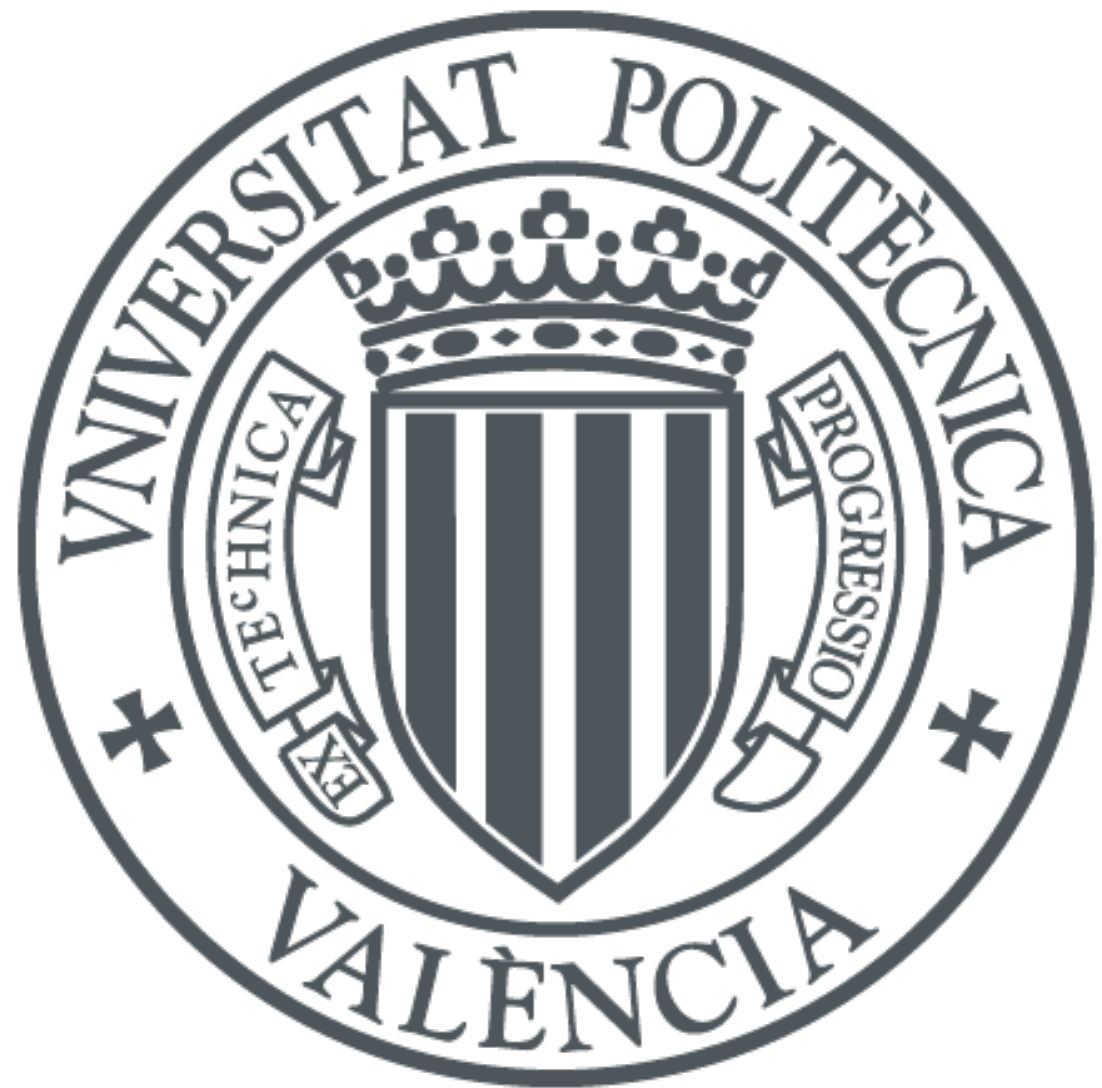

The final publication is available at

https://doi.org/10.1002/cctc.201901681

Copyright John Wiley \& Sons

Additional Information

This is the peer reviewed version of the following article: Y. Peng, J. Albero, H. García, ChemCatChem 2019, 11, 6384, which has been published in final form at https://doi.org/10.1002/cctc.201901681. This article may be used for non-commercial purposes in accordance with Wiley Terms and Conditions for Self-Archiving. 


\title{
Surface silanation of hybrid benzidinium lead perovskite and its influence on the photocatalytic activity
}

\author{
Yong Peng, ${ }^{a}$ Josep Albero, ${ }^{a}$ and Hermenegildo García ${ }^{a *}$
}

Instituto Universitario de Tecnología Química CSIC-UPV, Universitat Politècnica de València, Avda. De los Narajos s/n 46022, Valencia (Spain).

Corresponding: hgarcia@qim. upv. es; Te1: +34 963877807

\section{Abstract}

Surface coating of benzidinium lead iodide perovskite has been successfully accomplished by silanation, obtaining samples with average thickness from 2 to 6 $\mathrm{nm}$ as revealed by transmission electron microscopy. The obtained silica-coated hybrid perovskites exhibit enhanced hydrophobic character and, therefore, increased stability against moisture. However, its photocatalytic activity towards the cis-to-trans isomerization of stilbene diminishes as a function of the coating thickness, although a high activity for this photocatalytic reaction is still observed.

\section{Introduction}

Hybrid organic lead perovskites have drastically modified the landscape of photovoltaic cells by reaching very high efficiencies over $23 \%,{ }^{1}$ overcoming the values that were achieved with dye sensitized solar cells. ${ }^{2}$ The operation mechanism 
of photovoltaic cells has many fundamental steps in common with photocatalytic transformations, including light absorption, charge separation and carrier migration to the surface. ${ }^{3-5}$ However, in contrast to the impact that hybrid organic perovskites have had in the realm of solar cells, the lack of stability has precluded the use of this hybrid perovskites in photocatalysis. ${ }^{6,7}$

In an attempt to develop and explore optoelectronic properties of hybrid lead organic perovskites in photocatalysis, we have reported recently the preparation of $1 \mathrm{D}$ benzidinium lead iodide perovskite $\left(\mathrm{PbI}_{3} \mathrm{Bz}_{0.5}, \quad \mathrm{Bz}:{ }^{+} \mathrm{NH}_{3}-\mathrm{C}_{6} \mathrm{H}_{4}-\mathrm{C}_{6} \mathrm{H}_{4}-\mathrm{NH}_{3}{ }^{+}\right)$, (ref) the rationale for the preparation of this hybrid perovskite was to show the possibility to modulate optoelectronic properties of the material by proper selection of the organic ammonium cation. ${ }^{8-10}$ In particular, biphenyl derivatives have been widely used in photoinduced electron transfer reactions due to the ability to stabilize radical ions. $^{11,12}$

It was expected therefore that benzidinium dications should favor charge separation in the hybrid perovskite. Moreover, due to the size of the benzidnium cation, the 3D structure of the most widely used methylammonium lead iodide changed to 1D. Single crystal characterization of this novel perovskite shows that the material is constituted by $\mathrm{PbI}_{6}$ octahedra sharing the faces, forming an array of parallel $\mathrm{PbI}_{6}$ chains, that are held by Coulombic and hydrogen bridge interactions with the diammonium organic cations, see Scheme 1 (a). The empty space between the chains is occupied in the single crystal material by solvent DMF molecules. Although $\mathrm{PbI}_{3}(\mathrm{Bz})_{0.5}$ exhibited some range of stability, the material was instable in the 
presence of moisture and other polar solvents.

a)

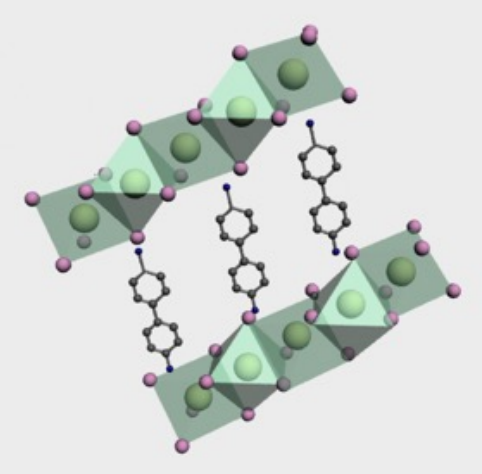

b)

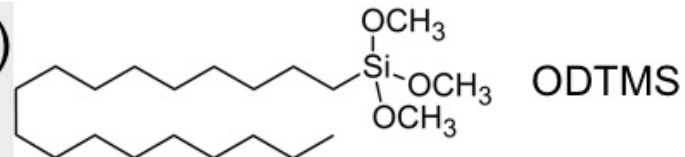

TEOS

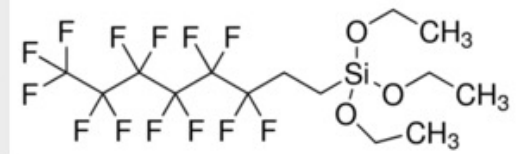

FTS

Scheme 1. (a) Structure of $\mathrm{PbI}_{3}(\mathrm{Bz})_{0.5}$ perovskite and (b) chemical structure of the three silylating reagents employed in this study (ODTMS, TEOS and FTS).

Continuing with this research line, in the present manuscript, we report the modification of $\mathrm{PbI}_{3}(\mathrm{Bz})_{0.5}$ perovskite surface by coating with silanating agents. There are sufficient data in the literature showing that surface silanation can protect materials by modifying the hydrophobic-hydrophilic properties of the surface. ${ }^{13-15}$ It will be shown below that capping of $\mathrm{PbI}_{3} \mathrm{BZD}_{0.5}$ can be successfully achieved by using conventional trialkoxy silane agents and that the photocatalytic activity of the silica-coated samples is still maintained even after coating of the hybrid perovskite surface with the thin silica layer.

\section{Results and discussion}

Surface modification by silane reagents is based on the presence of hydroxyl groups 
on the surface. ${ }^{16}$ Although ideally organic hybrid perovskites should not contain hydroxyl groups, contacting these materials with the ambient moisture results in a strong water adsorption that eventually has been proposed to replace $\mathrm{I}^{-}$ions by hydroxyl groups with concomitant evolution of HI. In fact, the presence of adsorbed water has been proposed as the main cause for the observed deterioration of photovoltaic efficiency in perovskite solar cells upon exposure to moist ambient. ${ }^{17,}$ ${ }^{18}$ The leading hypothesis of the present study is that also for $\mathrm{PbI}_{3} \mathrm{BZ}_{0.5}$ some hydroxyl groups could be present on the perovskite surface due to environmental humidity, ${ }^{19}$ and these hydroxyl groups would react with the silylating reagents forming a thin silica coating on the hybrid perovskite crystals. Herein, silanation was carried out using trimethoxy (octadecy1) silane (ODTMS), as we11 as tetra ethyl orthosilacate (TEOS) and triethoxy perfluorodecyl silane (FTS) as illustrated in Scheme 1 (b). The reaction was carried out under inert atmosphere by suspending the hybrid perovskite in a toluene solution at $50{ }^{\circ} \mathrm{C}$ containing the desired silylating reagent. These conditions are compatible with the preservation of the structure of the hybrid perovskite.

According to the thermogravimetric analysis (see Figure S1 in supporting information) and in situ XRD patterns, monitoring the crystalline structure at different temperatures (Figure S2 in supporting information) the hybrid perovskite crystallinity is maintained in the coating process. On the contrary, higher temperatures or the use of other solvents can cause change in the crystal phase or even decomposition of the hybrid perovskite. It was observed, however, that the XRD 
pattern of pristine $\mathrm{PbI}_{3} \mathrm{BZ}_{0.5}$ changes after the silanation step. Figure 1 shows a comparison between the XRD patterns of the hybrid benzidinium perovskite before and after silanation together with the XRD pattern of benzidnium perovskite exposed to hexane for $7 \mathrm{~h}$. This change in the XRD pattern was already observed in the related work, (ref) and it was attributed to the change in the 1D structure of hybrid perovskite crystals caused by the removal of DMF molecules in the intracrystalline space due to the dissolution of DMF in the organic solvents, such as hexane or toluene. The same hypothesis can be assumed here, since the material after silanation is still a crystalline material and the XRD patterns exactly match with the patterns derived from sample that presumably has lost the DMF molecules (Figure $1 \mathrm{~b}$ ). It should be commented that the quality of the crystals after silanation is not enough to perform structural elucidation by single crystal XRD.

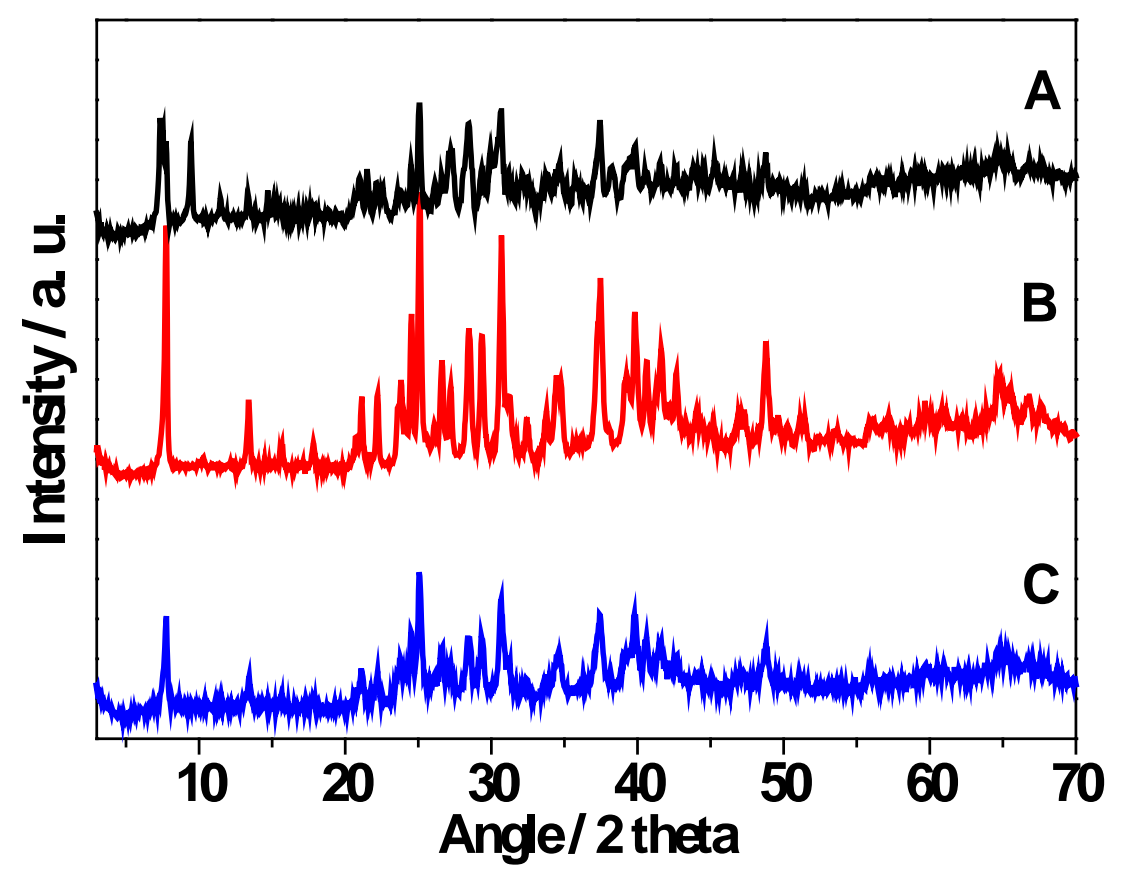


Figure 1. X-ray diffraction patterns of $\mathrm{PbI}_{3} \mathrm{BZD}_{0.5}$ (a), $\mathrm{PbI}_{3} \mathrm{BZD}_{0.5}$ after dispersion in hexane for $7 \mathrm{~h}$ (b) and silica-coated $\mathrm{PbI}_{3} \mathrm{BZD}_{0.5}$ after silanation with ODTMS (c).

Preservation of the optoelectronic properties of the silica-coated hybrid perovskite was accessed by diffuse reflectance UV-vis absorption spectroscopy presented in Figure 2. Only minor changes in the absorption onset of the hybrid perovskite was recorded after silica coating. Specifically, the absorption onset showed a small red-shift after silanation from $431 \mathrm{~nm}$ to $434 \mathrm{~nm}$. Accordingly, the Tauc plots showed a minor bandgap change from 2.78 to $2.76 \mathrm{eV}$. The small shift means after silanation, the hybrid perovskite held the optical properties of the parent material and the negligible changes in the absorption profile may arise from the organic-inorganic interaction at the interlayer.



Figure 2. Diffuse reflectance UV-Vis spectra absorption of $\mathrm{PbI}_{3} \mathrm{BZD}_{0.5}$ (black) and 
silica-coated $\mathrm{PbI}_{3} \mathrm{BZD}_{0.5}$ after silanation with ODTMS (red). Inset: Tauc plot of the corresponding $\mathrm{PbI}_{3} \mathrm{BZD}_{0.5}$ (black) and silica-coated $\mathrm{PbI}_{3} \mathrm{BZD}_{0.5}$ after silanation with OCTMS (red) with intercept used to calculate $\mathrm{E}_{\mathrm{g}}$ (dashed blue lines).

FTIR spectroscopy allows following the changes in the functional groups of the hybrid perovskite after silanation. Figure 3 shows the FTIR spectra of the samples prepared in this study. Initially, the FTIR spectrum of $\mathrm{PbI}_{3} \mathrm{BZD}_{0.5}$ shows a very broad vibration band from 3250 till $2250 \mathrm{~cm}^{-1}$ due to the acid X-H (X: N and 0) bonds present in the organic cation. Upon silanation, the presence of new vibration corresponding to $\mathrm{Si}-\mathrm{OH}$ silanol groups at $3500 \mathrm{~cm}^{-1}$ as well as $\mathrm{C}-\mathrm{H}$ stretching vibrations at $2950 \mathrm{~cm}^{-1}$ and $2916 \mathrm{~cm}^{-1}$ were clearly visible, as well as the characteristic Si-0 at about 850 $\mathrm{cm}^{-1}$.

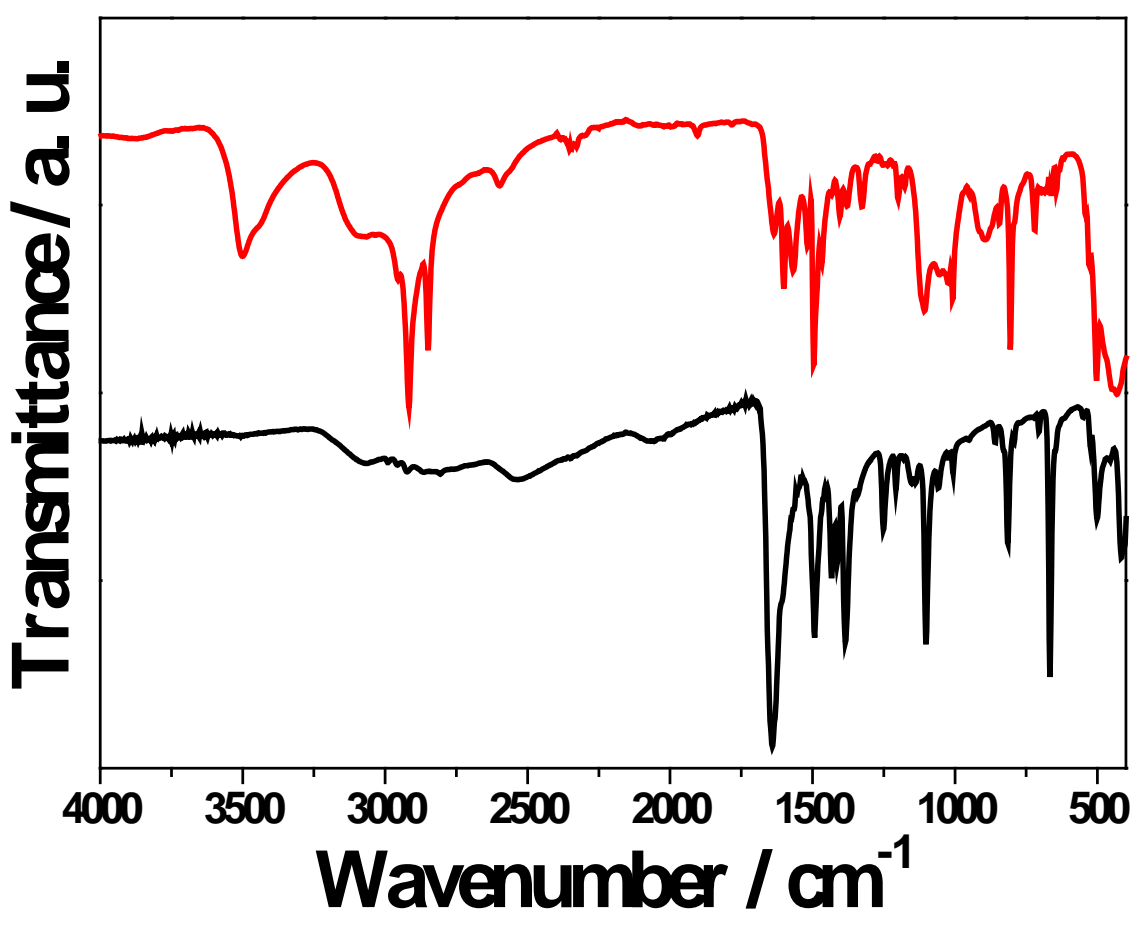


Figure 3. FTIR spectra of parent $\mathrm{PbI}_{3} \mathrm{BZD}_{0.5}$ (black) and silica-coated $\mathrm{PbI}_{3} \mathrm{BZD}_{0.5}$ after silanation with ODTMS (red).

High resolution XP spectra of $\mathrm{C} 1 \mathrm{~s}, 01 \mathrm{~s}, \mathrm{Si} 2 \mathrm{p}$ and $\mathrm{Pb} 4 \mathrm{f}$ peaks and the best deconvolution into individual components are shown in Figure 4. As it can be observed there, the XPS C1s peak (Figure 4 a) shows the presence of three main components attributed to $\mathrm{C}=\mathrm{C} \quad(284.6 \mathrm{eV}, \quad$ blue $), \quad \mathrm{C}-0 \quad(285.6 \mathrm{eV}, \quad$ orange $)$ and $\mathrm{C}-\mathrm{Si} \quad(283.1 \mathrm{eV}$, green). percentages???? The $01 \mathrm{~s}$ spectrum (Figure $4 \mathrm{~b}$ ) presents a main component attributed to 0 atoms single bonded to aliphatic $\mathrm{C}$ appearing at $532.2 \mathrm{eV}$, as wel1 as two smaller components related to 0 double bonded to sp2 $\mathrm{C}$ and $\mathrm{Si}-0$ bonds at 527.9 and $529.5 \mathrm{eV}$, respectively. The Si 2p spectrum (Figure $4 \mathrm{c}$ ) shows a main component related to the $\mathrm{Si}-\mathrm{C}$ bond from silane groups located at $102.2 \mathrm{eV}$, although a second component centered at $103.8 \mathrm{eV}$ assigned to $\mathrm{Si}-0$ can be also seen. The presence of - $\mathrm{OH}$ groups in the $\mathrm{Pb}$ surface cannot be clearly distinguished in the $\mathrm{Pb}$ 4f $7 / 2$ spectrum because of the overlap of the $\mathrm{Pb}^{+2}$ bonded to $\mathrm{I}^{-}$signal at $138.1 \mathrm{eV}$. 

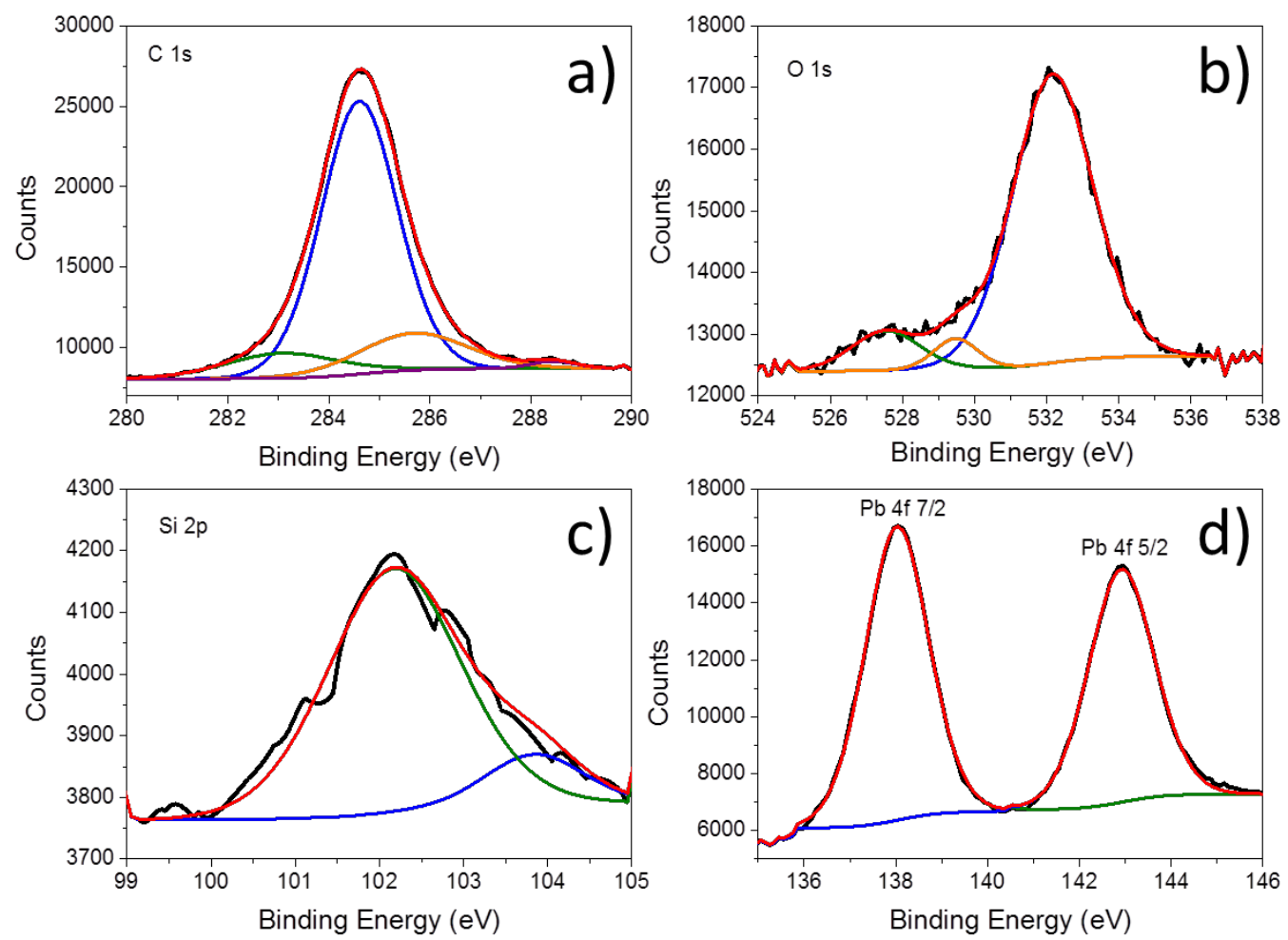

Figure 4. High resolution XPS peaks for $\mathrm{C} 1 \mathrm{~s}$ (a), 01s (b) Si 2p (c) and $\mathrm{Pb} 4 \mathrm{f}$ (d) recorded for silica-coated $\mathrm{PbI}_{3} \mathrm{BZD}_{0.5}$ perovskite, using ODTMS as reagent. The best deconvolution into individual components for each peak is also shown.

Solid-state ${ }^{29} \mathrm{Si}$ NMR spectroscopy was was recorded for the silica coated $\mathrm{PbI}_{3} \mathrm{BZD}_{0.5}$ sample. The spectrum is presented as Figure S3 in supporting Information. Peaks at $-49,-59$ and $-67 \mathrm{ppm}$ were recorded and attributed to $\mathrm{T}^{3}, \mathrm{~T}^{2}$ and $\mathrm{T}^{1} \mathrm{Si}$ atoms in the silane groups, confirming the presence of these moieties in the hybrid perovskite. Thus, all the available spectroscopic data are in agreement with the presence of a silica coating on the material surface.

In fact, the presence of the silica coating on the hybrid perovskite crystals could 
be clearly observed in HRTEM that also allows us determining the thickness of the silica layer. Samples with different average thickness were prepared in the case of silica-coated $\mathrm{PbI}_{3} \mathrm{BZ}_{0.5}$ perovskite by performing the silanation step using different OCTMS to perovskite mass ratios in the silanation step. Figure 5 shows representative HRTEM images of silica-coated $\mathrm{PbI}_{3} \mathrm{BZD}_{0.5}$ perovskite with different thickness. Elemental mapping by EDS (see Figure S4 in the supporting information) confirmed the presence of Si completely coating the perovskite crystals surface. EDX analysis on the surface and at the center of a sample revealed that the Si proportion at the edges is higher than the amount at the center for the silica capped perovskite (see Figure S5 in supporting information). This location of the silica layer agrees with the lighter contrast of the thin layers on the crystals observed in TEM. It should be noted that HRTEM images show that the thickness of the layers is not constant throughout the particle and changes depending on the area monitored were observed. Figure $\mathbf{S 6}$ in the supporting information shows the silica thickness average determined by measuring a statistical relevant number of samples as a function of the concentration of ODTMS used in the preparation of these samples.
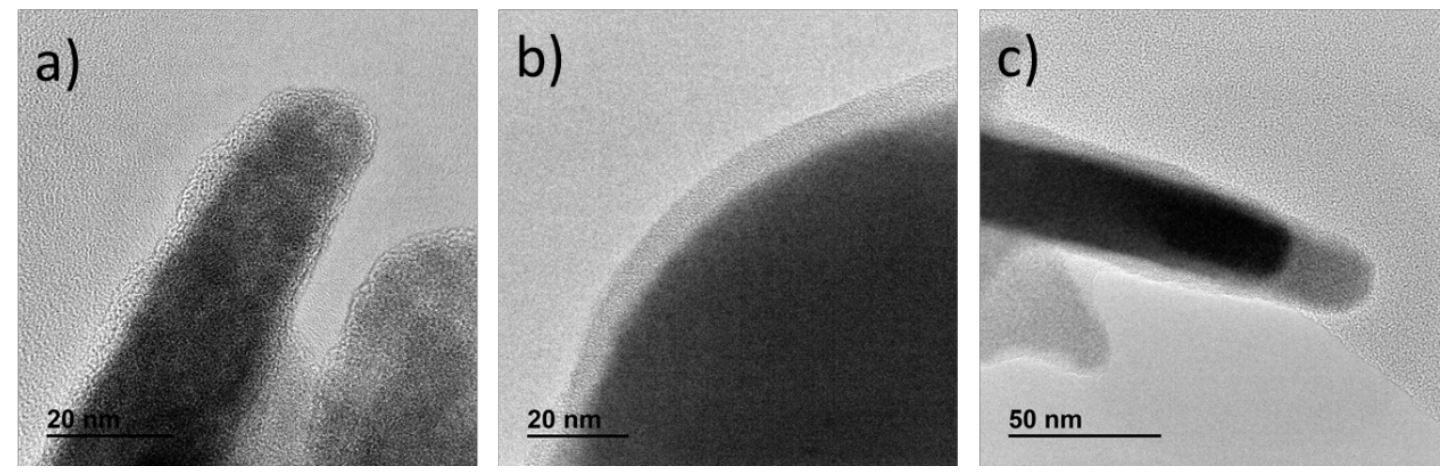
Figure 5. HRTEM images of silica-coated $\mathrm{PbI}_{3} \mathrm{BZD}_{0.5}$ perovskite with $1: 0.5$ (a), 1:1.5 (b) and 1:5 (c) ODTMS/perovskite mass ratio.

It should be commented that we tried to perform also surface salilation for the most common methylammonium lead perovskite with iodine or bromine ions using ODTMS as silylating agent, but the silanation failed in all cases as revealed by HRTEM (see Figure S7 in supporting information). This failure could be attributed to lack of a sufficient density of hydroxyl groups on the surface of these hybrid methylammonium perovskites. On the contrary, $\mathrm{PbI}_{3} \mathrm{BZD}_{0.5}$ perovskite was successfully coated not only with ODTMS, but a1so with TEOS and FTS (see Figure S8 in supporting information), pointing out to a general methodology for coating hybrid perovskites, particularly the one containing benzidinium organic ligands considered here.

Regarding the photocatalytic activity of $\mathrm{PbI}_{3} \mathrm{BZD}_{0.5}$, it was shown that this hybrid

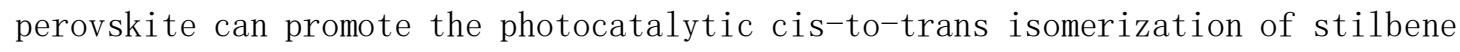
(Scheme 2) (ref). It was, therefore, of interest to determine if after capping with the silica layer the hybrid perovskite is still active to promote this photocatalytic reaction. Figure 6 (a) shows the temporal evolution of the photocatalytic cis-to-trans isomerization as function of the different silanating agents (TEOS, ODTMS and FTS). As it can be seen there, cis-to-trans isomerization occurs in the presence of the silica-capped perovskites, independently of the silylating reagent used to form the coating. It was, however, observed that the photocatalytic activity 
respect to the uncoated material decreased after coating. These results can be easily interpreted assuming that the surface of free parent perovskite is free to interact with cis-stilbene, which undergoes photoindiced electron transfer isomerization. The presence of the porous silica layer introduces some diffusing limitations to

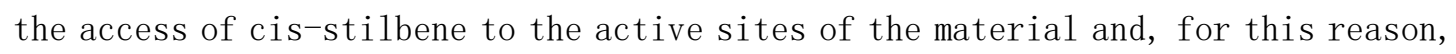
the photocatalytic reaction becomes slower. It is worth noticing that the photocatalytic activity of the different coated perovskites did not present drastic differences among them under the present reaction conditions. This could indicate either that the porosity of the silica layers is similar or that the photocatalytic isomerization occurs on the external surface.

In order to further investigate the influence of the silica coating thickness in the photocatalytic reaction the temporal evolution of the photocatalytic cis-to-trans isomerization of stilbene was studied for silica-coated samples of different average thickness. The results are presented in Figure 6 (b). It seems however that the thickness of the layer in the range from 2 to $5 \mathrm{~nm}$ screened in the present study do not make additional influence on the activity.
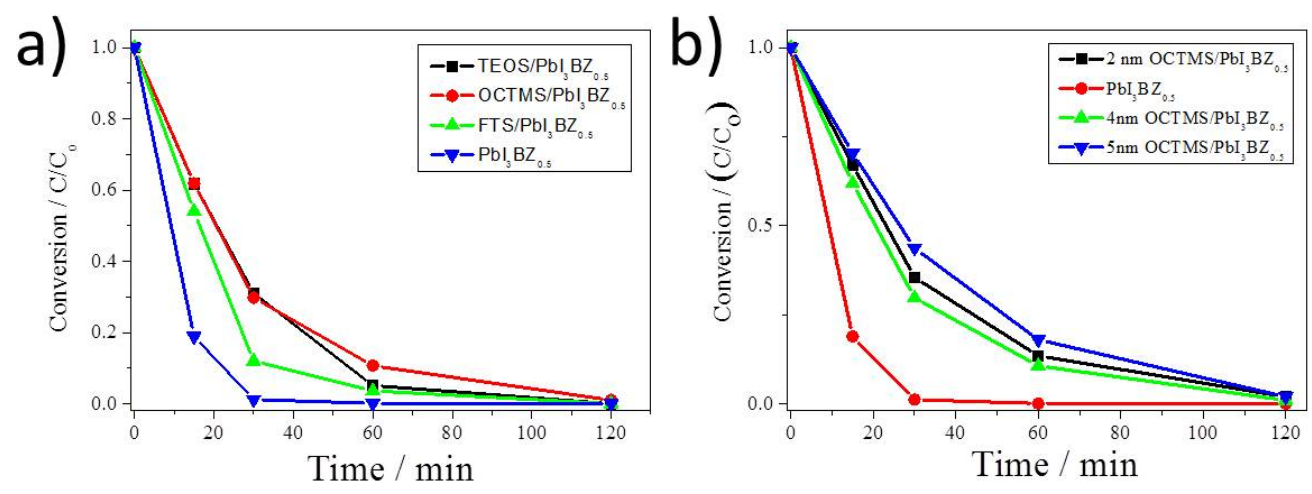
Figure 6. (a) Photocatalytic cis-to-trans isomerization of stilbene with using as photocatalysts the parent $\mathrm{PbI}_{3} \mathrm{BZD}_{0.5}$ perovskite or silica-coated samples prepared using TEOS, OCTMS and FTS silylating reagents. (b) Influence of the thickness or the photocatalytic cir-to-trans stilbene isomerization using parent $\mathrm{PbI}_{3} \mathrm{BZD}_{0.5}$ or silica-coated samples prepared using ODTMS as silylating reagent. Reaction conditions: XXX.

\section{Conclusion}

The present study has shown the possibility to coat the external surface of the hybrid organic lead perovskite $\mathrm{PbI}_{3} \mathrm{BZD}_{0.5}$ by silanation with different silylating reagents obtaining layers below $6 \mathrm{~nm}$. The silanation process depends on the density of hydroxyls groups on the surface of the hybrid perovskite. Although the reaction rate was somewhat lower using the silica coating, the photocatalytic activity of the silica-coated perovskites was still remarkable. Considering that silanation is a we11-known approach to modify the properties of the materials, the present study opens the door for the control of the photocatalytic properties of perovskites, particularly those that influence their stability in different solvents.

\section{References}

1. Q. Jiang, Y. Zhao, X. Zhang, X. Yang, Y. Chen, Z. Chu, Q. Ye, X. Li, Z. Yin and J. You, Nature Photonics, 2019.

2. K. Kakiage, Y. Aoyama, T. Yano, K. Oya, J.-i. Fujisawa and M. Hanaya, Chemical Communications, 2015, 51, 15894-15897.

3. R. Saravanan, F. Gracia and A. Stephen, in Nanocomposites for Visible Light-induced Photocatalysis, eds. M. M. Khan, D. Pradhan and Y. Sohn, Springer International Publishing, Cham, 2017, pp. 19-40. 
4. J. Bisquert, D. Cahen, G. Hodes, S. Rühle and A. Zaban, The Journal of Physical Chemistry B, 2004, 108, 8106-8118.

5. P. V. Kamat, Accounts of Chemical Research, 2017, 50, 527-531.

6. C. C. Boyd, R. Cheacharoen, T. Leijtens and M. D. McGehee, Chem Rev, 2019, 119, 3418-3451.

7. N. Aristidou, C. Eames, I. Sanchez-Molina, X. Bu, J. Kosco, M. S. Islam and S.

A. Haque, Nature Communications, 2017, 8, 15218.

8. D. Cortecchia, S. Neutzner, A. R. Srimath Kandada, E. Mosconi, D. Meggiolaro, F. De Angelis, C. Soci and A. Petrozza, J Am Chem Soc, 2017, 139, 39-42.

9. N. Kawano, M. Koshimizu, Y. Sun, N. Yahaba, Y. Fujimoto, T. Yanagida and K. Asai, The Journal of Physical Chemistry C, 2014, 118, 9101-9106.

10. J. Albero and H. García, Journal of Materials Chemistry C, 2017, 5, 4098-4110.

11. A. Ronlan, J. Coleman, 0. Hammerich and V. D. Parker, Journal of the American Chemical Society, 1974, 96, 845-849.

12. M. R. Talipov, A. Boddeda, Q. K. Timerghazin and R. Rathore, The Journal of Physical Chemistry C, 2014, 118, 21400-21408.

13. P. A. Zapata, Y. Huang, M. A. Gonzalez-Borja and D. E. Resasco, Journal of Catalysis, 2013, 308, 82-97.

14. J. Bu and H. -K. Rhee, Catalysis Letters, 2000, 65, 141-145.

15. Y. Kuwahara, K. Maki, Y. Matsumura, T. Kamegawa, K. Mori and H. Yamashita, The Journal of Physical Chemistry C, 2009, 113, 1552-1559.

16. W. Yoshida, R. P. Castro, J.-D. Jou and Y. Cohen, Langmuir, 2001, 17, 5882-5888.

17. T. A. Berhe, W. - N. Su, C. -H. Chen, C. -J. Pan, J. -H. Cheng, H. -M. Chen, M. -C. Tsai, L. -Y. Chen, A. A. Dubale and B.-J. Hwang, Energy \& Environmental Science, 2016, 9, 323-356.

18. G. Niu, X. Guo and L. Wang, Journal of Materials Chemistry A, 2015, 3, 8970-8980.

19. B. Cheng, T. - Y. Li, P. - C. Wei, J. Yin, K. -T. Ho, J. R. D. Retamal, 0. F. Mohammed and J.-H. He, Nature Communications, 2018, 9, 5196. 